\title{
Accurate Estimation of Electromagnetic Wave Extinction through Foliage
}

\author{
Feinian Wang \\ Radiation Laboratory, EECS Department \\ The University of Michigan \\ Ann Arbor, MI 48109-2122, USA \\ feinianw@eecs.umich.edu
}

\author{
Kamal Sarabandi \\ Radiation Laboratory, EECS Department \\ The University of Michigan \\ Ann Arbor, MI 48109-2122, USA \\ saraband@eecs.umich.edu
}

\begin{abstract}
In this paper a new statistical wave propagation (SWAP) model is introduced to model the wave propagation behavior through long distance forested environments. It divides the forest into statistically identical blocks along the wave propagation direction. By applying the existing single scattering wave theory model to one representative block of forest, it can pre-compute and store the statistical properties of the forest which can then be reused to compute the total power at the receiver. The computation intensity is significantly reduced while the modeling accuracy is enhanced. Three sets of simulation experiments are conducted to validate the SWAP model and the results are presented.

Keywords-wave propagation; path-loss; single scattering; multiple scattering; forested environment
\end{abstract}

\section{INTRODUCTION}

The accurate modeling of wave propagation behavior through forested environments is of great interest for remote sensing applications. Existing foliage propagation models treat the forest as an effective lossy dielectric medium which predict an exponential increasing of path-loss with respect to the propagation distance within the forest. Such model captures only the coherent power of the signal which comes from the mean electromagnetic field propagating through the forest and neglects the scattered signal that could be redirected towards forward direction after scattered by the randomly distributed scatterers inside the forest, such as branches and leaves. At UHF and higher, the size of the scatterers becomes a significant portion of or larger than a wavelength and therefore can cause significant scattering. For long distance wave propagation, such incoherent power tends to dominate the overall received power after a certain distance because of the exponential-decaying behavior of the coherent power.

In order to capture the contribution from the incoherent power, two different approaches, namely the radiative transfer theory and the wave theory, may be used [1]. The radiative transfer approach is not suitable for the forested environment which contains large scatterers such as trunks and branches, due to the difficulties in determining the phase and extinction matrices. The second approach, based on wave theory, uses Foldy's approximation [2] to estimate the coherent mean-field along the direction of propagation and uses this mean-field as the incident wave illuminating each constituent particle inside the forest. The scattered field from each scatterer can then be calculated and added coherently. The advantage of such approach is its inherent high fidelity since it allows the use of realistic-looking tree structures for the forest model that can significantly affect the scattered field statistics. This approach, known as the single scattering wave theory model, has been successfully implemented and verified for a number of applications [3, 4, 5]. However, for high scatterer density, multiple scattering effects become important. Recent study has shown that path-loss calculation using single scattering may provide an overestimation $[5,6]$. Another issue of concern is the computation time. Keeping track of all scatterers and scattering components over a long propagation distance is computationally prohibitive. This difficulty is circumvented in this paper by treating the forest as statistically homogeneous medium along the direction of wave propagation. Based on this assumption, a new Statistical WAve Propagation (SWAP) model is presented and is shown to provide a computationally efficient solution for estimation of electromagnetic wave extinction in forested environment over long distances. Such model also improves the accuracy over the existing single scattering model, due to the capability of including partial multiple scattering effects through a recursive approach.

\section{SWAP MODEL DESCRIPTION}

Figure 1 shows the scenario for long distance wave propagation through a forest under a horizontal incident wave. The statistically homogeneous forest is divided into blocks with the same width along the direction of wave propagation. These blocks are assumed to be statistically identical and independent of each other. Therefore, the wave propagation behavior of each block is identical and the field computation can be localized into one block. This provides the statistical properties of the wave propagation over a short distance which can effectively be used to calculate the statistical field properties over long distances. The width of each block is carefully selected to ensure capturing all scattering mechanisms at the same time to keep the numerical simulation tractable. By reusing the statistical properties computed for a typical block, one can calculate the incoherent power radiated from each block to the receiver. Summing all the incoherent power generated by each block and adding to the coherent power which can be calculated from the incident wave attenuated by the forest, the total power at the receiver, and 
hence the path-loss or wave extinction, is obtained. The incoherent power generated by each block is also attenuated by the successive blocks before reaching the receiver. The precomputed forest statistical properties depend on different forest attributes such as tree structures and densities, as well as signal parameters such as frequencies and polarizations.

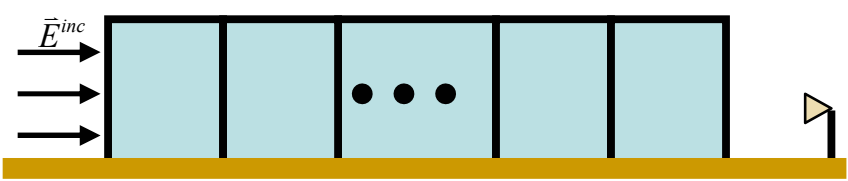

Figure 1: Long distance forest divided into statistically identical and independent blocks.

To compute the incoherent scattering components using the statistical parameters estimated from one typical forest block, the total field at the surfaces of each block is split into the sum of the mean-field and the fluctuating field, as shown in Figure 2. That is, at the output surface of the $\mathrm{j}^{\text {th }}$ block, the field distribution can be written as

$$
\vec{E}_{j}\left(\vec{r}^{\prime}\right)=\left\langle\vec{E}_{j}\left(\vec{r}^{\prime}\right)\right\rangle+\widetilde{E}_{j}\left(\vec{r}^{\prime}\right),
$$

where $\left\langle\widetilde{E}_{j}\left(\vec{r}^{\prime}\right)\right\rangle=0$. The fluctuating field $\widetilde{E}_{j}\left(\vec{r}^{\prime}\right)$, indicated by the ripple line in Figure 2, is due to the scattered field from the random scatterers within the $\mathrm{j}^{\text {th }}$ block. According to Huygens' principle, the equivalent currents associated with $\widetilde{E}_{j}\left(\vec{r}^{\prime}\right)$ act as secondary radiating sources and generate the fluctuating field, $\widetilde{E}_{j}(\vec{r})$, at the receiver. Similarly, the fluctuating field at the receiver caused by scatterers within other forest blocks can be calculated and added coherently to compute the total fluctuating field, i.e.

$$
\widetilde{E}(\vec{r})=\sum_{j} \widetilde{E}_{j}(\vec{r}) \cdot
$$

The total field at the receiver is therefore

$$
\vec{E}(\vec{r})=\langle\vec{E}(\vec{r})\rangle+\widetilde{E}(\vec{r}),
$$

where the coherent mean-field $\langle\vec{E}(\vec{r})\rangle$ can be estimated using Foldy's approximation. The average power received by the receiver contains coherent and incoherent components and is given by

$$
\left\langle|\vec{E}(\vec{r})|^{2}\right\rangle=|\langle\vec{E}(\vec{r})\rangle|^{2}+\left\langle|\widetilde{E}(\vec{r})|^{2}\right\rangle,
$$

where the incoherent power $\left\langle|\widetilde{E}(\vec{r})|^{2}\right\rangle$ includes contributions from each individual forest block according to equation (2), i.e.

$$
\left\langle|\widetilde{E}(\vec{r})|^{2}\right\rangle=\sum_{j}\left\langle\left|\widetilde{E}_{j}(\vec{r})\right|^{2}\right\rangle \cdot
$$

Here the statistical independence of different blocks is assumed.

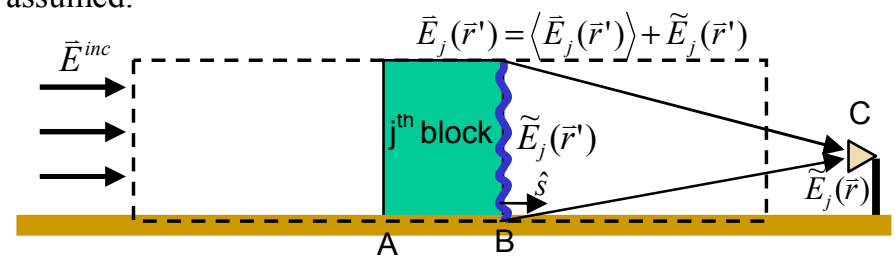

Figure 2: Fluctuating filed generates incoherent power at the receiver.
The fluctuating field distribution at the output surface of the $\mathrm{j}^{\text {th }}$ block, $\widetilde{E}_{j}\left(\vec{r}^{\prime}\right)$, is computed statistically. It is assumed that the coefficient of variation, defined by the ratio of the standard deviation to the mean of $\vec{E}_{j}\left(\vec{r}^{\prime}\right)$, is the same for all forest blocks. Such parameter is a function of the random medium property of the forest, and can be estimated from a typical block and reused for other blocks. The standard deviation of $\widetilde{E}_{j}\left(\vec{r}^{\prime}\right)$ is in fact equal to the standard deviation of $\vec{E}_{j}\left(\vec{r}^{\prime}\right)$ since $\left\langle\widetilde{E}_{j}\left(\vec{r}^{\prime}\right)\right\rangle=0$. Once the coefficient of variation is known, the standard deviation of $\widetilde{E}_{j}\left(\vec{r}^{\prime}\right)$ can be obtained by multiplying it with the mean-field $\left\langle\vec{E}_{j}\left(\vec{r}^{\prime}\right)\right\rangle$. Assuming complex random variable $\widetilde{E}_{j}\left(\vec{r}^{\prime}\right)$ is Gaussian distributed, a random number generator samples of $\widetilde{E}_{j}\left(\vec{r}^{\prime}\right)$ can be generated easily. The coefficient of variation for a forest block is computed by applying the single scattering wave theory model in conjunction with Monte-Carlo simulations to this block. The mean-field $\left\langle\vec{E}_{j}\left(\vec{r}^{\prime}\right)\right\rangle$ is the average of the total field at the output surface under an aggregation of different excitations at the input surface of the $\mathrm{j}^{\text {th }}$ block, such as the coherent meanfield, and the scattered wave from the $(\mathrm{j}-1)^{\text {th }}$ block, or equivalently, the equivalent surface currents of $\widetilde{E}_{j-1}\left(\vec{r}^{\prime \prime}\right)$ at the output surface of the $(j-1)^{\text {th }}$ block. The mean-field component at the output surface of the $\mathrm{j}^{\text {th }}$ block corresponding to the coherent mean-field illumination, $\left\langle\vec{E}_{j}\left(\vec{r}^{\prime}\right)\right\rangle^{\text {Foldy }}$, can be estimated using Foldy's approximation. The component corresponding to illumination of the scattered wave from previous block can be calculated by $\overline{T_{1}}\left(\vec{r}^{\prime}, \vec{r}^{\prime \prime}\right) \cdot \widetilde{E}_{j-1}\left(\vec{r}^{\prime \prime}\right)$, where $\overline{\overline{T_{1}}}\left(\vec{r}^{\prime}, \vec{r}^{\prime \prime}\right)$ is the transfer matrix between the field distributions at the input and output surfaces of the $j^{\text {th }}$ block. It relays the scattered field from scatterers inside the $(\mathrm{j}-1)^{\text {th }}$ block to the mean-field component at the output surface of the $\mathrm{j}^{\text {th }}$ block. $\overline{\overline{T_{1}}}\left(\vec{r}^{\prime}, \vec{r}^{\prime \prime}\right)$ is considered a homogeneous statistical parameter to be reused for each individual block, and can be generalized for all the previous forest blocks before the $\mathrm{j}^{\text {th }}$ block, therefore accounts for all the scattering effects from previous blocks. In this case, the overall mean-field at the output surface of the $j^{\text {th }}$ block is given by

$$
\left\langle\vec{E}_{j}\left(\vec{r}^{\prime}\right)\right\rangle=\left\langle\vec{E}_{j}\left(\vec{r}^{\prime}\right)\right\rangle^{\text {Foldy }}+\sum_{1}^{j-1} \overline{\overline{T_{n}}}\left(\vec{r}^{\prime}, \vec{r}^{\prime \prime}\right) \cdot \widetilde{E}_{j-n}\left(\vec{r}^{\prime \prime}\right),
$$

where $\widetilde{E}_{j-n}\left(\vec{r}^{\prime \prime}\right)$ is the fluctuating field on the output surface of the $(j-n)^{\text {th }}$ block, which represents all the scattering effects involving the scatterers inside the $(\mathrm{j}-\mathrm{n})^{\text {th }}$ block, and also acts as the input radiating source to the $\mathrm{j}^{\text {th }}$ block. $\overline{\overline{T_{n}}}\left(\vec{r}^{\prime}, \vec{r}^{\prime \prime}\right)$ is just the generalization of $\overline{\bar{T}}_{1}\left(\vec{r}^{\prime}, \vec{r}^{\prime \prime}\right)$. Equation (6) constitutes a recursive approach where the effects of scattering from all previous blocks are accounted for through the mean-field $\left\langle\vec{E}_{j}\left(\vec{r}^{\prime}\right)\right\rangle$, which, after multiplying with the coefficient of variation parameter, adds in the scattering effect from the $\mathrm{j}^{\text {th }}$ 
block. Once the fluctuating field, $\widetilde{E}_{j}\left(\vec{r}^{\prime}\right)$, is obtained, the incoherent power generated from each block can be computed, where the statistical spatial correlation coefficient $C\left(\vec{r}_{1}{ }^{\prime}, \vec{r}_{2}{ }^{\prime}\right)$ is also needed. The detailed formulation for computation of the incoherent power at the receiver is provided in the next section. Figure 3 sketches the algorithm flowchart of the SWAP model. $\mathrm{M}_{\mathrm{mn}}$ is the signal attenuation coefficient estimated according to Foldy's approximation. $\sigma / \mu$ is the coefficient of variation of the output surface field distribution of a typical block. As the flowchart indicates, the total power is the summation of the coherent power, which comes from the direct mean-field computed by Foldy's approximation, and the total incoherent power computed recursively using the $\overline{\bar{T}}$ matrix and other statistical parameters of the forest. The existing single scattering wave theory model [4] can be applied to a typical forest block to compute all necessary statistical parameters.

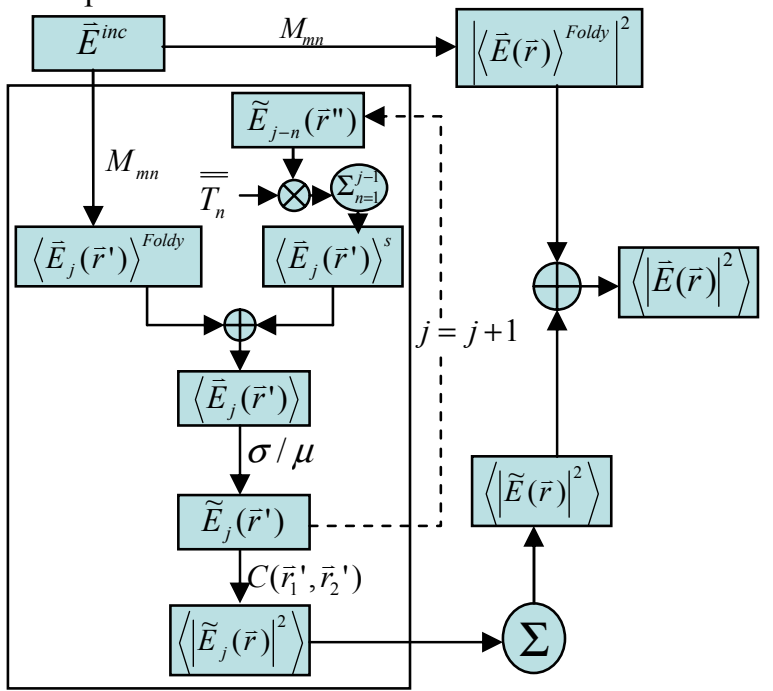

Figure 3: Algorithm flowchart to calculate total power at receiver.

\section{COMPUTATION OF INCOHERENT POWER}

The incoherent power results from the fluctuation field radiated from each block of forest to the receiver. Based on the field equivalence principle, the fluctuation field at the receiver radiated from the $\mathrm{j}^{\text {th }}$ block of forest in Figure 2 is given by

$$
\widetilde{E}_{j}(\vec{r})=-\iint_{B} \nabla^{\prime} \times \overline{\bar{G}}\left(\vec{r}, \vec{r}^{\prime}\right) \cdot 2 \widetilde{J}_{m}\left(\vec{r}^{\prime}\right) d s^{\prime},
$$

where

$$
\widetilde{J}_{m}\left(\vec{r}^{\prime}\right)=\widetilde{E}_{j}\left(\vec{r}^{\prime}\right) \times \hat{s}
$$

is the equivalent magnetic surface current and $\hat{S}$ is the outward unit vector normal to $\boldsymbol{B}$, the output surface of the $\mathrm{j}^{\text {th }}$ forest block. Ground effect is taken into account using approximate image theory [7]. The factor of 2 reflects the equivalent magnetic current sources backed by a planar perfect electric conductor over surface $\boldsymbol{B} . \overline{\bar{G}}\left(\vec{r}, \vec{r}^{\prime}\right)$ is the dyadic Green's function of a medium having a permittivity equal to the effective dielectric constant of the forest. Theoretically, the surface $\boldsymbol{B}$ should be infinite in extent; however, since the fluctuation field is mainly confined to the forest vertical dimension the electric field computation for calculating $\widetilde{J}_{m}$ is limited to the vertical extent of the forest. Now suppose surface $\boldsymbol{B}$ is $d$ meters tall and $a$ meters wide, then the incoherent power can be calculated as

$$
\begin{aligned}
& \left\langle\left|\widetilde{E}_{j}(\vec{r})\right|^{2}\right\rangle=\left\langle\widetilde{E}_{j}(\vec{r}) \cdot \widetilde{E}_{j}(\vec{r})^{*}\right\rangle \\
& \left.\left.=\left\langle\left(-\int_{0}^{d} \int_{-a / 2}^{a / 2} \nabla^{\prime} \times \overline{\bar{G}}\left(\vec{r}, \vec{r}_{1}{ }^{\prime}\right) \cdot 2 \widetilde{J}_{m}\left(\vec{r}_{1}{ }^{\prime}\right)\right] d s_{1}{ }^{\prime}\right) \cdot\left(-\int_{0}^{d} \int_{-a / 2}^{a / 2} \nabla^{\prime} \times \bar{G}\left(\vec{r}, \vec{r}_{2}{ }^{\prime}\right) \cdot 2 \widetilde{J}_{m}\left(\vec{r}_{2}{ }^{\prime}\right)\right] d s_{2}{ }^{\prime}\right)^{*}\right\rangle \\
& =\frac{1}{4 \pi^{2}} \int_{0}^{d} \int_{-a / 2}^{a / 2} \int_{0}^{d} \int_{-a / 2}^{a / 2} \frac{e^{j k_{e} R_{1}-j k_{e 2}^{*} R_{2}}\left(j k_{e 1} R_{1}-1\right)\left(-j k_{e 2}^{*} R_{1}-1\right)}{R_{1}^{3} R_{2}^{3}} \\
& \cdot\left[\left\langle\widetilde{E}_{j z}\left(\vec{r}_{1}^{\prime}\right) \cdot \widetilde{E}_{j z}\left(\vec{r}_{2}^{\prime}\right)^{*}\right\rangle\left(z-z_{1}^{\prime}\right)\left(z-z_{2}{ }^{\prime}\right)\right. \\
& +\left\langle\widetilde{E}_{j z}\left(\vec{r}_{1}^{\prime}\right) \cdot \widetilde{E}_{j y}\left(\vec{r}_{2}{ }^{\prime}\right)^{*}\right\rangle\left(z-z_{1}{ }^{\prime}\right)\left(y-y_{2}{ }^{\prime}\right)+\left\langle\widetilde{E}_{j y}\left(\vec{r}_{1}^{\prime}\right) \cdot \widetilde{E}_{j z}\left(\vec{r}_{2}{ }^{\prime}\right)^{*}\right\rangle\left(y-y_{1}{ }^{\prime}\right)\left(z-z_{2}{ }^{\prime}\right) \\
& +\left\langle\widetilde{E}_{j y}\left(\vec{r}_{1}{ }^{\prime}\right) \cdot \widetilde{E}_{j y}\left(\vec{r}_{2}{ }^{\prime}\right)^{*}\right\rangle\left(y-y_{1}{ }^{\prime}\right)\left(y-y_{2}{ }^{\prime}\right)+\left\langle\widetilde{E}_{j y}\left(\vec{r}_{1}^{\prime}\right) \cdot \widetilde{E}_{j y}\left(\vec{r}_{2}{ }^{\prime}\right)^{*}\right\rangle\left(x-x_{1}{ }^{\prime}\right)\left(x-x_{2}{ }^{\prime}\right) \\
& \left.+\left\langle\widetilde{E}_{j z}\left(\vec{r}_{1}{ }^{\prime}\right) \cdot \widetilde{E}_{j z}\left(\vec{r}_{2}{ }^{\prime}\right)^{*}\right\rangle\left(x-x_{1}{ }^{\prime}\right)\left(x-x_{2}{ }^{\prime}\right)\right] d y_{1}{ }^{\prime} d z_{1}{ }^{\prime} d y_{2}{ }^{\prime} d z_{2}{ }^{\prime}
\end{aligned}
$$

where

$$
\begin{aligned}
& \left\langle\widetilde{E}_{p}\left(\vec{r}_{1}^{\prime}\right) \cdot \widetilde{E}_{q}\left(\vec{r}_{2}^{\prime}\right)^{*}\right\rangle=\operatorname{COV}\left[\widetilde{E}_{p}\left(\vec{r}_{1}^{\prime}\right), \widetilde{E}_{q}\left(\vec{r}_{2}^{\prime}\right)\right] . \\
& =C\left[\widetilde{E}_{p}\left(\vec{r}_{1}^{\prime}\right), \widetilde{E}_{q}\left(\vec{r}_{2}^{\prime}\right)\right] \sigma\left[\widetilde{E}_{p}\left(\vec{r}_{1}^{\prime}\right)\right] \sigma\left[\widetilde{E}_{q}\left(\vec{r}_{2}^{\prime}\right)\right]
\end{aligned}
$$

$\operatorname{COV}\left[\widetilde{E}_{p}\left(\vec{r}_{1}^{\prime}\right), \widetilde{E}_{q}\left(\vec{r}_{2}^{\prime}\right)\right]$ and $C\left[\widetilde{E}_{p}\left(\vec{r}_{1}^{\prime}\right), \widetilde{E}_{q}\left(\vec{r}_{2}^{\prime}\right)\right]$ represent the covariance and the normalized correlation function between the fluctuation field at $\vec{r}_{1}^{\prime}$ with polarization $p$ and the fluctuation field at $\vec{r}_{2}{ }^{\prime}$ with polarization $q \cdot \sigma\left[\widetilde{E}_{p}\left(\vec{r}_{1}^{\prime}\right)\right]$ is the standard deviation of the fluctuation field at $\vec{r}_{1}^{\prime}$ with polarization $p$, and is defined by

$$
\sigma\left[\widetilde{E}_{p}\left(\vec{r}_{1}^{\prime}\right)\right]=\sqrt{\left\langle\left|\widetilde{E}_{p}\left(\vec{r}_{1}^{\prime}\right)\right|^{2}\right\rangle}=\sqrt{\left\langle\left|\vec{E}_{p}\left(\vec{r}_{1}^{\prime}\right)\right|^{2}\right\rangle-\left|\left\langle\vec{E}_{p}\left(\vec{r}_{1}^{\prime}\right)\right\rangle\right|^{2}} .
$$

$k_{e}$ is the effective wave number and $R$ is the distance from $\vec{r}^{\prime}$ to $\vec{r}$. Both stationary phase approximation method [8] and numerical integration are employed in the computation.

\section{Simulation Results}

Three sets of simulation experiments are conducted to validate the SWAP model. For all experiments, the forest is generated based on a specific structure of red pine tree, which is about $8 \mathrm{~m}$ tall, with a trunk layer about $1.2 \mathrm{~m}$ high, and a crown diameter of approximately $5 \mathrm{~m}$. A block of forest $10 \mathrm{~m}$ long, $10 \mathrm{~m}$ wide, and $8 \mathrm{~m}$ tall is used for parameter estimation. Plane wave incidence is assumed. The receiver is located $1.5 \mathrm{~m}$ above the ground and moved to several spots along the direction of wave propagation. For frequencies considered in the simulations (f $<4 \mathrm{GHz}$ ), scattering from needles is negligible and ignored. All simulations are performed on a Linux workstation with a $2.4 \mathrm{GHz}$ processor and $1 \mathrm{~GB}$ memory. First, the comparison between the SWAP model and the existing single scattering wave theory propagation model is made by examining the path-loss due to the forest at $0.5 \mathrm{GHz}$ versus the propagation distance within a forest, which is $200 \mathrm{~m}$ long and $10 \mathrm{~m}$ wide and has a tree density of $0.05 / \mathrm{m}^{2}$. Multiple scattering effects, which are accounted for by the $\overline{\bar{T}}$ matrix in the SWAP model, is neglected. As shown in Figure 4(a), the 
SWAP model agrees very well with the "brute force" numerical result obtained from the single scattering model to within 2-3 dB. In addition, the new model provides the expected behavior of path-loss in foliage as a function of distance. The simulation time using SWAP model, about 200 seconds with pre-stored statistical parameters, is significantly lower than the single scattering model, about 30 hours. The one-time computation of the statistical parameters takes about 8 hours. Next, the SWAP model is applied to the forests with different tree densities at the same frequency $(0.5 \mathrm{GHz})$. Figure 4(b) shows the path-loss versus propagation distance for three different tree densities. As expected, larger tree density causes more attenuation and scattering, therefore more incoherent scattering power relative to the coherent power at the receiver. This is also the reason for the path-loss curve to bend at a shorter propagation distance. Whereas the mean-field decreases with increasing tree density monotonically, the total path-loss decreases for higher densities after the path-loss curve bends. Finally, to examine the multiple scattering effects, the $\overline{\bar{T}}$ matrix is included in the SWAP model. A tree density of $0.15 / \mathrm{m}^{2}$ is selected, where the multiple scattering effects are significant. Simulation frequency is chosen to be $0.5 \mathrm{GHz}$ to reduce the computation time. As shown in Figure 4(c), an obvious decrease of path-loss is observed at the ranges where incoherent power dominates. Since multiple scattering also contributes to the incoherent power, it increases the overall received power and hence reduces the path-loss through the forest.

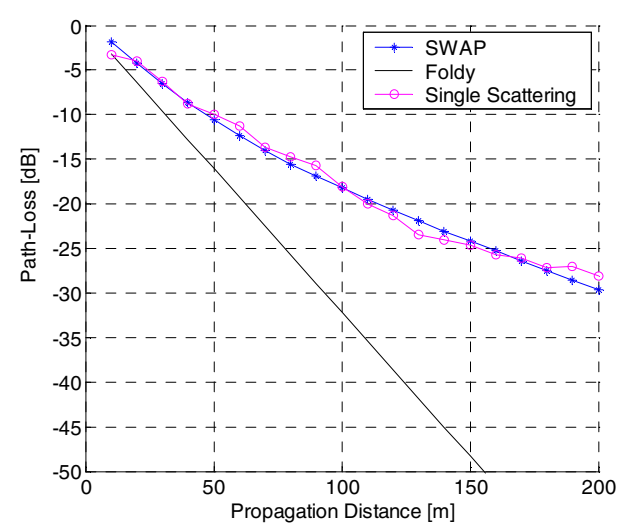

(a)

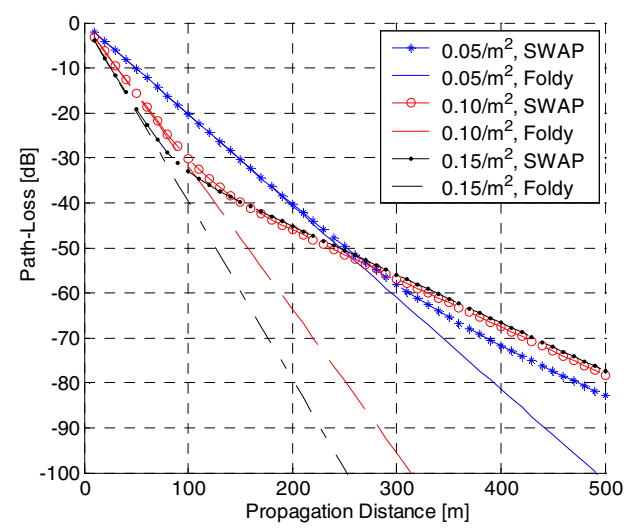

(b)

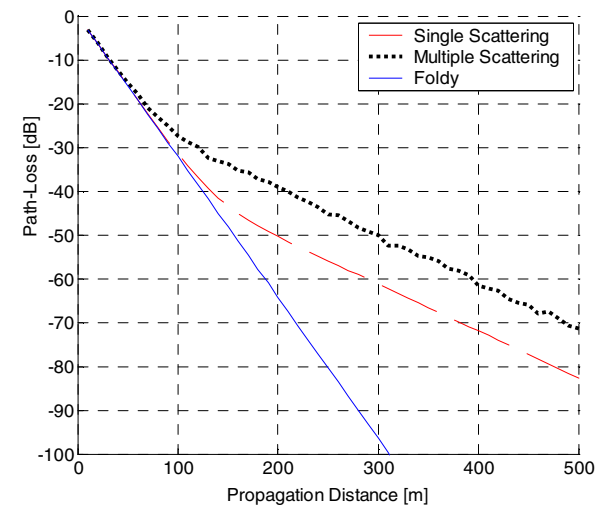

(c)

Figure 4: Validation of SWAP model, (a) comparison between SWAP model and single scattering model; (b) comparison between SWAP model applied at different tree densities; (c) examination of multiple scattering effects.

\section{CONCLUSION}

A new statistical wave propagation (SWAP) model is implemented to estimate the wave extinction through long distance forests. Three sets of simulation experiments conducted in this paper validated the model and showed that both significantly reduced computation time and enhanced modeling accuracy are achieved by the SWAP model.

\section{REFERENCES}

[1] A. Ishimaru, Wave Propagation and Scattering in Random Media, IEEE Press, New York, 1997.

[2] L. Tsang, J.A. Kong, and R.T. Shin, Theory of Microwave Remote Sensing, John Wieley \& Sons, New York, 1985.

[3] Y.C. Lin and K. Sarabandi, "A Monte Carlo Coherent Scattering Model for Forest Canopies Using FractalGenerated Trees," IEEE Trans. Geosci. Remote Sensing, vol. 37, No. 1, pp. 440-451, 1999.

[4] I. Koh and K. Sarabandi, "Polarimetric Channel Characterization of Foliage for Performance Assessment of GPS Receivers under Tree Canopies," IEEE Trans. Antennas Propagation, vol. 50, No. 5, pp. 713-726, 2002.

[5] F. Wang and K. Sarabandi, "An Enhanced Microwave and Millimeter-Wave Foliage Propagation Model," submitted to IEEE Trans. Antennas Propagation.

[6] I. Koh, F. Wang, and K. Sarabandi, "Estimation of Coherent Field Attenuation Through Dense Foliage Including Multiple Scattering," IEEE Trans. Geosci. Remote Sensing, vol. 41, No. 5, pp. 1132-1135, 2003.

[7] R.E. Collin and F.J. Zucker, Antenna Theory, McGrawHill, New York, 1969.

[8] C.A. Balanis, Antenna Theory: analysis and design, Second Edition, pp. 922-926, John Wiley \& Sons, Inc., New York, 1997. 